Mathematical Modelling and Analysis

Volume 21 Number 3, May 2016, 371-384

http://dx.doi.org/10.3846/13926292.2016.1172130

(c) Vilnius Gediminas Technical University, 2016
Publisher: Taylor\&Francis and VGTU

http://www.tandfonline.com/TMMA

ISSN: $1392-6292$

eISSN: 1648-3510

\title{
Pullback Attractor for a Non-Autonomous Generalized Cahn-Hilliard Equation with Biological Applications
}

Ning Duan

${ }^{a}$ School of Science, Jiangnan University

214122 Wuxi, China

E-mail: 123332453@qq. com

Received August 3, 2015; revised March 23, 2016; published online May 15, 2016

\begin{abstract}
In this paper, we consider a non-autonomous generalized Cahn-Hilliard equation with biological applications. It is shown that a pullback attractor of the equation exists when the external force has exponential growth.
\end{abstract}

Keywords: pullback attractor, generalized Cahn-Hilliard equation, asymptotic compactness.

AMS Subject Classification: 35B41; 35K35.

\section{Introduction}

The Cahn-Hilliard equation was proposed to describe phase separation phenomena in binary systems (see [2]). The well-known form of Cahn-Hilliard equation is written as follows:

$$
\frac{\partial u}{\partial t}+\Delta(\Delta u-f(u))=0
$$

where $u=u(x, t)$ is an unknown function representing the relative concentration of one species. Many papers have already been published to study the Cahn-Hilliard equation. For example, in [9,24], the problem of stability and long time behavior of solutions was studied; Gilardi et al. [14] considered the Cahn-Hilliard equation with dynamic boundary conditions; Cherfils et al. [6] investigated the Cahn-Hilliard equation in phase separation with the thermodynamically relevant logarithmic potentials; Schimperna [21] considered the global attractors for Cahn-Hilliard equations with nonconstant mobility. On the other hand, due to a wide application of a hyperbolic type of equations in humane society and in biology $[10,11]$. It would be logical and consistent to speculate about application and possible analysis of the hyperbolic CahnHilliard generalized equation. In [12,13], Galenko et al. have proposed to add the inertial term in order to model non-equilibrium decompositions caused by 
deep supercooling in certain glasses. For more results on hyperbolic CahnHilliard equation, we refer the reader to $[15,22]$ and the references therein.

Recently, Khain and Sander [16] proposed a generalized Cahn-Hilliard equation for biological applications:

$$
\frac{\partial u}{\partial t}-\frac{\partial^{2}}{\partial x^{2}}\left[\ln (1-q) \frac{\partial^{2} u}{\partial x^{2}}+F^{\prime}(u)\right]+\alpha u(u-1)=0 .
$$

Equation (1.1) is modelling cells which move, proliferate and interact via adhesion in wound healing and tumor growth. Here, $u$ is the local density of cells, $q$ is the adhesion parameter, $\alpha>0$ is the proliferation rate, $F$ is the local free energy. Furthermore,

$$
q=1-\exp \left(-\frac{J}{k_{B} T}\right),
$$

where $J$ corresponds to the interatomic interaction, $k_{B}$ is the Boltzmann's constant and $T$ is the absolute temperature, assumed constant.

In [7], for simplicity, Cherfils, Miranville and Zelik set all physical constants equal to 1 and solved the problem in the higher space dimension (in two space dimensions, the equation models, e.g., the clustering of malignant brain tumor cells, see $[7,16])$, i.e., they studied asymptotic behavior the generalized CahnHilliard equation

$$
\frac{\partial u}{\partial t}+\Delta^{2} u-\Delta f(u)+g(u)=0
$$

endowed with Neumann boundary conditions, where $g(s)=s(s-1)$ and $f(s)=$ $s^{3}-s$.

Remark 1. Equation (1.2) can also been seen as a generalized diffusion model for growth and dispersal in a population model (see [8]). Suppose $u(x, t)$ represents the population density, $D$ is a proportionality constant, $f(u)$ represents the energy density which this volume would have in a homogeneous composition, and the other terms reprensent the energy density which is a function of local composition and which clearly will be significant in non-homogeneous states. $F[u]=\int_{\Omega}\left[f(u)+\frac{1}{2} k(\nabla u)^{2}+\cdots\right] d x$ is the total energy function, the variational derivative $\frac{\delta F}{\delta u}$ defines a potential $\mu(u)$. Based on Fick's law, the flux $J$ is proportional to the gradient of $\mu$. Then,

$$
\frac{\partial u}{\partial t}=-\operatorname{div} J=-k D \Delta^{2} u+\operatorname{div}\left(D f^{\prime \prime}(u) \operatorname{grad} u\right)
$$

Finally, if we incorporate the dynamics (or reaction terms) $G(u)$, we obtain

$$
\frac{\partial u}{\partial t}=-D k \Delta^{2} u+D A \Delta u+D B \Delta u^{3}+G(u)
$$

which is similar to equation (1.2). There's also some other papers concerned with this diffusion model, for example $[18,25]$ and so on.

The study of pullback attractor for nonautonomous infinite dimensional dynamical systems has attracted much attention and made fast progress in 
recent years; see for instance, [4,23]. Recently, Caraballo et al. [5] introduced the notion of the pullback $\mathcal{D}$-attractor for nonautonomous dynamical systems and gave a general method to prove the existence of pullback $\mathcal{D}$-attractor. In [17], Li and Zhong proposed the concept of norm-to-weak continuous process and proved the existence of pullback attractors for the nonautonomous reactiondiffusion equation.

There's also some papers studied pullback attractor for non-autonomous fourth order nonlinear diffusion equations. For example, the authors in [20] considered the existence of pullback attractor for a nonautonomous modified Swift-Hohenberg equation when its external force has exponential growth. Caraballo and Colucci [3] proved the existence of a pullback attractor for the initial boundary(Dirichlet boundary conditions) value problem of the following non-autonomous fourth order evolution equation

$$
\frac{\partial u}{\partial t}+\varepsilon^{2} \frac{\partial^{4} u}{\partial x^{4}}-\frac{1}{2} W^{\prime \prime}\left(\frac{\partial u}{\partial x}\right) \frac{\partial^{2} u}{\partial x^{2}}=f(x, t),
$$

which arise in the field of phase transitions and elasticity theory.

In this article, suppose that $h \in L_{l o c}^{2}\left(\mathbb{R}, L^{2}(0,1)\right)$ is an external forcing term. We are concerned with the following non-autonomous generalized CahnHilliard equation:

$$
\frac{\partial u}{\partial t}+\frac{\partial^{4} u}{\partial x^{4}}-\frac{\partial^{2}}{\partial x^{2}} f(u)+g(u)=h(x, t), \quad x \in(0,1), \quad t \in[\tau, \infty),
$$

where $g(s)=s(s-1)$ and $f(s)=s^{3}-s$. Equation (1.3) is supplied with the boundary value conditions

$$
u(x, 0)=u(x, 1)=\frac{\partial u}{\partial x}(x, 0)=\frac{\partial u}{\partial x}(x, 1)=0
$$

and the initial condition

$$
u(x, \tau)=u_{\tau}(x), \quad x \in(0,1) .
$$

The paper is organized as follows. In Section 2, we recall some abstract results on pullback attractors and give the main result. In Section 3, we prove the existence of pullback attractor for problem (1.3)-(1.5).

Throughout this paper, we denote $(\cdot, \cdot)$ as the inner product of $L^{2}(0,1)$ and $\|\cdot\|$ as the induced norm. $\|\cdot\|_{X}$ denotes the norm of a Banach space $X$. For simplicity, we denote $\|\cdot\|_{L^{p}(0,1)}$ by $\|\cdot\|_{p}$, respectively.

\section{Preliminary}

In this section, we give some basic definitions and results on the existence of pullback attractor. Suppose that $X$ is a complete metric space and $\{U(t, \tau)\}=$ $\{U(t, \tau): t \geq \tau, \tau \in \mathbb{R}\}$ is a two-parameter family of mappings act on $X$ : $U(t, \tau): X \rightarrow X, t \geq \tau, \tau \in \mathbb{R}$.

Definition 1 [see [17]]. A two-parameter family of mappings $\{U(t, \tau)\}$ is said to be norm-to-weak continuous process in $X$ if 
- $U(t, s) U(s, \tau)=U(t, \tau), \quad \forall t \geq s \geq \tau$,

- for all $\tau \in \mathbb{R}, U(t, \tau)=I d$ is the identity operator,

- $U(t, \tau) x_{n} \rightarrow U(t, \tau) x$, if $x_{n} \rightarrow x$ in $X$.

Let $B$ be a bounded subset of $X$. The Kuratowski measure of noncompactness $\alpha(B)$ of $B$ is defined by

$\alpha(B)=\inf \{\delta>0 \mid B$ has a finite open cover of sets of diameter $\leq \delta\}$.

Suppose $\mathcal{D}$ is a nonempty class of parameterised sets $\hat{\mathcal{D}}=\{D(t): t \in \mathbb{R}\} \subset$ $B(X)$.

Definition 2 [see [17]]. A process $\{U(t, \tau)\}$ is called pullback $\omega$-D-limit compact if for any $\varepsilon>0$ and $\hat{D} \in \mathcal{D}$, there exists a $\tau_{0}(t, \hat{\mathcal{D}} \leq t$ such that $\alpha\left(U_{\tau \geq \tau_{0}} U(t, \tau) D(\tau)\right) \leq \varepsilon$.

Definition 3 [see [17]]. The family $\hat{A}=\{A(t): t \in \mathbb{R}\} \subset B(X)$ is said to be a pullback $\mathcal{D}$-attractor for $U(t, \tau)$ if

- for all $t \in \mathbb{R}, A(t)$ is compact,

- $\hat{A}$ is invariant, i.e., $U(t, \tau) A(\tau)=A(\tau), \quad \forall t \geq \tau$,

- $\hat{A}$ is pullback $\mathcal{D}$-attracting, i.e.,

$$
\lim _{\tau \rightarrow-\infty} \operatorname{dist}(U(t, \tau) D(\tau), A(t))=0, \quad \forall \hat{\mathcal{D}} \in \mathcal{D}, t \in \mathbb{R}
$$

- if $\{C(t)\}_{t \in \mathbb{R}}$ is another family of closed attracting sets, then $A(t) \subset C(t)$ for all $t \in \mathbb{R}$.

In the following, we give the result on the existence of pullback $\mathcal{D}$-attractor for nonautonomous systems which can be seen in [17].

Lemma 1. Let $\{U(t, \tau)\}_{\tau \leq t}$ be a norm-to-weak continuous process such that $\{U(t, \tau)\}_{\tau \leq t}$ is pullback $\omega$-D-limit compact. If there exists a family of pullback $\mathcal{D}$-absorbing sets $\{B(t): t \in \mathbb{R}\} \in \mathcal{D}$, i.e., for any $t \in \mathbb{R}$ and $\hat{D} \in \mathcal{D}$ there is a $\tau_{0}(t, \hat{D}) \leq t$ such that $U(t, \tau) D(\tau) \subset B(t)$ for all $\tau \leq \tau_{0}$. Then, there is a pullback $\mathcal{D}$-attractor $\{A(t): t \in \mathbb{R}\}$ and

$$
A(t)=\bigcap_{s \leq t} \overline{\bigcup_{\tau \leq s} U(t, \tau) D(\tau)} .
$$

In order to study pullback attractor for the non-autonomous problem (1.3)(1.5), we set $A=\frac{\partial^{4}}{\partial x^{4}}$ and $\lambda$ be the first eigenvalues of $A$. For the external force $h$, we assume that there is a non-negative constant $\beta$ such that

$$
\|h(t)\|^{2} \leq \beta e^{\alpha|t|}, \quad 0 \leq \alpha<\frac{\lambda}{81} .
$$


Then, simple calculation shows that

$$
\begin{aligned}
& G_{1}(t):=\int_{-\infty}^{t} e^{\lambda s}\|h(s)\|^{2} d s<\infty, \quad \forall t \in \mathbb{R}, \\
& G_{2}(t):=\int_{-\infty}^{t} \int_{-\infty}^{s} e^{\lambda s}\|h(s)\|^{2} d s d t<\infty, \quad \forall t \in \mathbb{R}, \\
& \int_{-\infty}^{t} e^{-8 \lambda r}\left\{G_{1}(r)\right\}^{6} d r<\infty, \quad \int_{-\infty}^{t} e^{-\frac{8}{7} \lambda r}\left\{G_{1}(r)\right\}^{\frac{15}{7}} d r<\infty, \quad \forall t \in \mathbb{R} .
\end{aligned}
$$

By a standard method, we can prove that for every $\tau \in \mathbb{R}$ and $u_{\tau} \in H_{0}^{2}(0,1)$, there exists a unique solution $u(x, t) \in C\left([\tau, \infty), H_{0}^{2}(0,1)\right)$ (see e.g. $\left.[7,19]\right)$. Moreover, the solution $u(x, t)$ is continuous with respect to the initial value condition $u_{\tau}$ in the space $H_{0}^{2}(0,1)$. In order to construct a process $\{U(t, \tau)\}$ for problem $(1.3)-(1.5)$, we define $U(t, \tau): H_{0}^{2}(0,1) \rightarrow H_{0}^{2}(0,1)$ by $U(t, \tau) u_{\tau}$. Thus, the process $\{U(t, \tau)\}$ is a norm-to-weak continuous process in the space $H_{0}^{2}(0,1)$.

Our main purpose is to study the existence of pullback attractor for problem (1.3)-(1.5) by employing the techniques in $[17,20]$. The main difficulties for treating the problem (1.3)-(1.5) are caused by the nonlinear terms. Since the term $g(s)=s(s-1)$ is a polynomial of order 2 on $s \in \mathbb{R}$, it is difficult to deal with this term on the process of a prior estimates for problem (1.3)-(1.5).

Employing the techniques in [19], we introduce the inverse operator $\left(-\frac{\partial^{2}}{\partial x^{2}}\right)^{-1}$, which is a positive self-adjoint operator, to handle the nonlinear term $g(u)$; Due to the two terms $\frac{\partial^{2}}{\partial x^{2}} f(u)$ and $g(u)$ in (1.3), the estimates are delicate. We impose the exponential growth conditions (2.1) on the external forcing term $h(x, t)$ to overcome this difficulty.

Now, we give the main result of this paper, which will be proved in the next section.

Theorem 1. The process corresponding to problem (1.3)-(1.5) possesses a $u$ nique pullback $\mathcal{D}$-attractor in the space $H_{0}^{2}(0,1)$.

Throughout this paper, the letter $c$ is a generic positive constant that may change from line to line even if in the same inequality.

\section{Proof of Theorem 1}

Now, we study the existence of pullback attractors for non-autonomous problem (1.3)-(1.5). First of all, we introduce the definition of the norm $\|\cdot\|_{-1}$, which will be used in this section.

Proposition 1 [see [1]]. For $v \in L^{2}(0,1)$, the solution $\xi$ (denote by $N(v)$ ) of the Dirichlet problem

$$
\left\{\begin{aligned}
-\frac{\partial^{2} \xi}{\partial x^{2}} & =v, \quad \text { in }(0,1), \\
\xi & =0, \quad x=0,1 .
\end{aligned}\right.
$$


Furthermore, the norm $\left\|\frac{\partial^{2} \xi}{\partial x^{2}}\right\|$ is equivalent to the canonical norm $\|\xi\|_{2}$. Hence, we can denote $\|q\|_{-1}=\left\|\frac{\partial N(q)}{\partial x}\right\|$.

Then, we derive uniform estimates of solutions for problem (1.3)-(1.5), which are necessary for proving the existence of absorbing set of $\{u(t, \tau)\}$ associated with the problem.

Lemma 2. Consider the problem (1.3)-(1.5), for all $t \geq \tau$, we have

$$
\begin{gathered}
\|u(t)\|_{-1}^{2}+\|u(t)\|^{2} \leq e^{-\lambda(t-\tau)}\left(\left\|u_{\tau}\right\|_{-1}^{2}+\left\|u_{\tau}\right\|^{2}\right)+\frac{M}{\lambda}+\frac{4}{\lambda} e^{-\lambda t} G_{1}(t) \\
\int_{\tau}^{t} e^{\lambda s}\left\|\frac{\partial^{2}}{\partial x^{2}} u(s)\right\|^{2} d s \leq[1+\lambda(t-\tau)] e^{\lambda \tau}\left(\left\|u_{\tau}\right\|_{-1}^{2}+\left\|u_{\tau}\right\|^{2}\right) \\
+\frac{2 M}{\lambda} e^{\lambda t}+\frac{4}{\lambda} G_{1}(t)+G_{2}(t)
\end{gathered}
$$

Proof. Multiplying equation (1.3) by $\left(-\frac{\partial^{2}}{\partial x^{2}}\right)^{-1} u(t)$, integrating it over $(0,1)$, we derive that

$$
\begin{aligned}
& \frac{1}{2} \frac{d}{d t}\|u(t)\|_{-1}^{2}+\left\|\frac{\partial}{\partial x} u(t)\right\|^{2}+\|u(t)\|_{4}^{4}+\|u(t)\|_{-1}^{2} \\
= & -\left(h(t),\left(-\frac{\partial^{2}}{\partial x^{2}}\right)^{-1} u(t)\right)+\|u(t)\|^{2}+\left([u(t)]^{2},\left(-\frac{\partial^{2}}{\partial x^{2}}\right)^{-1} u(t)\right) \\
\leq & \|h(t)\|\left\|\left(-\frac{\partial^{2}}{\partial x^{2}}\right)^{-1} u(t)\right\|+\|u(t)\|^{2}+\|u(t)\|_{4}^{2}\left\|\left(-\frac{\partial^{2}}{\partial x^{2}}\right)^{-1} u(t)\right\| \\
\leq & \frac{1}{\lambda}\|h(t)\|\|u(t)\|+\|u(t)\|^{2}+\frac{1}{\lambda}\|u(t)\|_{4}^{2}\|u(t)\| \\
\leq & \frac{\|h(t)\|^{2}}{\lambda}+\frac{1}{16}\|u(t)\|_{4}^{4}+\left(\frac{1}{4 \lambda}+1+\frac{4}{\lambda^{2}}\right)\|u(t)\|^{2} \\
\leq & \frac{\|h(t)\|^{2}}{\lambda}+\frac{1}{8}\|u(t)\|_{4}^{4}+\frac{M_{1}}{2},
\end{aligned}
$$

where $M_{1}=8\left(\frac{1}{4 \lambda}+1+\frac{4}{\lambda^{2}}\right)^{2}$ is a positive constant. Multiplying equation (1.3) by $u$, integrating it over $(0,1)$, we obtain

$$
\begin{aligned}
& \frac{1}{2} \frac{d}{d t}\|u(t)\|^{2}+\left\|\frac{\partial^{2}}{\partial x^{2}} u(t)\right\|^{2}+3\left\|u(t) \frac{\partial}{\partial x} u(t)\right\|^{2} \\
& =\left\|\frac{\partial}{\partial x} u(t)\right\|^{2}+\|u(t)\|^{2}-\left([u(t)]^{2}, u(t)\right)+(h(t), u(t)) \\
& \leq\left(\frac{1}{4}\left\|\frac{\partial^{2}}{\partial x^{2}} u(t)\right\|^{2}+\|u(t)\|^{2}\right)+\|u(t)\|^{2}+\left(\frac{1}{16}\|u(t)\|_{4}^{4}+4\|u(t)\|^{2}\right) \\
& \quad+\left(\frac{\|h(t)\|^{2}}{\lambda}+\frac{\lambda}{4}\|u(t)\|^{2}\right)
\end{aligned}
$$




$$
\begin{aligned}
& \leq \frac{1}{4}\left\|\frac{\partial^{2}}{\partial x^{2}} u(t)\right\|^{2}+6\|u(t)\|^{2}+\frac{1}{16}\|u(t)\|_{4}^{4}+\frac{\|h(t)\|^{2}}{\lambda}+\frac{\lambda}{4}\|u(t)\|^{2} \\
& \leq \frac{1}{2}\left\|\frac{\partial^{2}}{\partial x^{2}} u(t)\right\|^{2}+6\|u(t)\|^{2}+\frac{\|h(t)\|^{2}}{\lambda}+\frac{1}{16}\|u(t)\|_{4}^{4} \\
& \leq \frac{1}{2}\left\|\frac{\partial^{2}}{\partial x^{2}} u(t)\right\|^{2}+\frac{1}{8}\|u(t)\|_{4}^{4}+\frac{\|h(t)\|^{2}}{\lambda}+\frac{M_{2}}{2},
\end{aligned}
$$

where $M_{2}$ is a positive constant. Combining (3.1) and (3.2) together, we deduce that

$$
\begin{gathered}
\frac{d}{d t}\left(\|u(t)\|_{-1}^{2}+\|u(t)\|^{2}\right)+2\left\|\frac{\partial}{\partial x} u(t)\right\|^{2}+\left\|\frac{\partial^{2}}{\partial x^{2}} u(t)\right\|^{2}+\frac{3}{2}\|u(t)\|_{4}^{4} \\
+6\left\|u(t) \frac{\partial}{\partial x} u(t)\right\|^{2}+2\|u(t)\|_{-1}^{2} \leq M_{1}+M_{2}+\frac{4}{\lambda}\|h(t)\|^{2},
\end{gathered}
$$

that is

$$
\frac{d}{d t}\left(\|u(t)\|_{-1}^{2}+\|u(t)\|^{2}\right)+\lambda\left(\|u(t)\|_{-1}^{2}+\|u(t)\|^{2}\right) \leq M_{1}+M_{2}+\frac{4}{\lambda}\|h(t)\|^{2} .
$$

Multiplying (3.4) by $e^{\lambda(t-\tau)}$ and integrating it over $(\tau, t)$, we derive that

$$
\begin{aligned}
\|u(t)\|_{-1}^{2}+\|u(t)\|^{2} \leq & e^{-\lambda(t-\tau)}\left(\left\|u_{\tau}\right\|_{-1}^{2}+\left\|u_{\tau}\right\|^{2}\right) \\
& +\frac{M_{1}+M_{2}}{\lambda}+\frac{4}{\lambda} e^{-\lambda t} \int_{-\infty}^{t} e^{\lambda s}\|h(s)\|^{2} d s .
\end{aligned}
$$

Multiplying (3.5) by $e^{\lambda t}$ and integrating it over $(\tau, t)$, we deduce that

$$
\begin{gathered}
\int_{\tau}^{t} e^{\lambda s}\left(\|u(s)\|_{-1}^{2}+\|u(s)\|^{2}\right) d s \leq(t-\tau) e^{\lambda \tau}\left(\left\|u_{\tau}\right\|_{-1}^{2}+\left\|u_{\tau}\right\|^{2}\right) \\
+\frac{M_{1}+M_{2}}{\lambda^{2}} e^{\lambda t}+\frac{4}{\lambda} \int_{-\infty}^{t} \int_{-\infty}^{s} e^{\lambda r}\|h(r)\|^{2} d r d s
\end{gathered}
$$

Similarly, multiplying (3.3) by $e^{\lambda t}$ and integrating it over $(\tau, t)$, we get

$$
\begin{gathered}
\int_{\tau}^{t} e^{\lambda s}\left\|\frac{\partial^{2}}{\partial x^{2}} u(t)\right\|^{2} d s \leq e^{\lambda \tau}\left(\left\|u_{\tau}\right\|_{-1}^{2}+\left\|u_{\tau}\right\|^{2}\right)+\frac{M_{1}+M_{2}}{\lambda} e^{\lambda t} \\
+\lambda \int_{\tau}^{t} e^{\lambda s}\left(\|u(s)\|_{-1}^{2}+\|u(s)\|^{2}\right) d s+\frac{4}{\lambda} \int_{-\infty}^{t} e^{\lambda s}\|h(s)\|^{2} d s \\
\leq[1+\lambda(t-\tau)] e^{\lambda \tau}\left(\left\|u_{\tau}\right\|_{-1}^{2}+\left\|u_{\tau}\right\|^{2}\right)+\frac{2\left(M_{1}+M_{2}\right)}{\lambda} e^{\lambda t} \\
+\int_{-\infty}^{t} \int_{-\infty}^{s} e^{\lambda r}\|h(r)\|^{2} d r d s+\frac{4}{\lambda} \int_{-\infty}^{t} e^{\lambda s}\|h(s)\|^{2} d s .
\end{gathered}
$$

Then, the proof is completed. 
Lemma 3. Consider the problem (1.3)-(1.5), for all $t \geq \tau$, we have

$$
\begin{aligned}
& \left\|\frac{\partial^{2}}{\partial x^{2}} u(t)\right\|^{2} \leq c\left[( 1 + ( t - \tau ) + \frac { 1 } { t - \tau } ) e ^ { - \lambda ( t - \tau ) } \left[\left\|u_{\tau}\right\|_{-1}^{2}+\left\|u_{\tau}\right\|^{2}+\left\|u_{\tau}\right\|_{-1}^{18}\right.\right. \\
& \left.\quad+\left\|u_{\tau}\right\|^{18}+\left\|u_{\tau}\right\|_{-1}^{\frac{30}{7}}+\left\|u_{\tau}\right\|^{\frac{30}{7}}\right]+\left(1+\frac{1}{t-\tau}\right)+\left(1+\frac{1}{t-\tau}\right) e^{-\lambda t} \\
& \left.\quad \times\left[G_{1}(t)+G_{2}(t)\right]+e^{-\lambda t} \int_{\tau}^{t} e^{-8 \lambda s}\left[G_{1}(s)\right]^{9} d s+e^{-\lambda t} \int_{\tau}^{t} e^{-\frac{8}{7} \lambda s}\left[G_{1}(s)\right]^{\frac{15}{7}} d s\right] .
\end{aligned}
$$

Proof. Using Nirenberg's inequality, we have

$$
\begin{aligned}
& \|u(t)\|_{8} \leq c\left\|\frac{\partial^{4}}{\partial x^{4}} u(t)\right\|^{\frac{3}{32}}\|u(t)\|^{\frac{29}{32}}, \quad\left\|\frac{\partial^{2}}{\partial x^{2}} u(t)\right\|_{4} \leq c\left\|\frac{\partial^{4}}{\partial x^{4}} u(t)\right\|^{\frac{9}{16}}\|u(t)\|^{\frac{7}{16}}, \\
& \|u(t)\|_{\infty} \leq c\left\|\frac{\partial^{4}}{\partial x^{4}} u(t)\right\|^{\frac{1}{8}}\|u(t)\|^{\frac{7}{8}}, \quad\left\|\frac{\partial}{\partial x} u(t)\right\|_{4} \leq c\left\|\frac{\partial^{4}}{\partial x^{4}} u(t)\right\|^{\frac{5}{16}}\|u(t)\|^{\frac{11}{16}}, \\
& \|u(t)\|_{4} \leq c\left\|\frac{\partial^{4}}{\partial x^{4}} u(t)\right\|^{\frac{1}{16}}\|u(t)\|^{\frac{15}{16}} .
\end{aligned}
$$

Multiplying (1.3) by $\frac{\partial^{4}}{\partial x^{4}} u(t)$, integrating it over $(0,1)$, using Nirenberg's inequality, we derive that

$$
\begin{aligned}
& \frac{1}{2} \frac{d}{d t}\left\|\frac{\partial^{2}}{\partial x^{2}} u(t)\right\|^{2}+\left\|\frac{\partial^{4}}{\partial x^{4}} u(t)\right\|^{2}=\left(\frac{\partial^{2}}{\partial x^{2}}[u(t)]^{3}-\frac{\partial^{2}}{\partial x^{2}} u(t)-[u(t)]^{2}\right. \\
& \left.+u(t)+h(t), \frac{\partial^{4}}{\partial x^{4}} u(t)\right)=\left(3[u(t)]^{2} \frac{\partial^{2}}{\partial x^{2}} u(t)+6 u(t)\left|\frac{\partial}{\partial x} u(t)\right|^{2}-\frac{\partial^{2}}{\partial x^{2}} u(t)\right. \\
& \left.-[u(t)]^{2}+h(t), \frac{\partial^{4}}{\partial x^{4}} u\right)+\left\|\frac{\partial^{2}}{\partial x^{2}} u\right\|^{2} \\
& \leq 3\|u(t)\|_{8}^{2}\left\|\frac{\partial^{2}}{\partial x^{2}} u(t)\right\|_{4}\left\|\frac{\partial^{4}}{\partial x^{4}} u(t)\right\|+6\|u(t)\|_{\infty}\left\|\frac{\partial}{\partial x} u(t)\right\|_{4}^{2}\left\|\frac{\partial^{4}}{\partial x^{4}} u(t)\right\| \\
& +\left\|\frac{\partial^{2}}{\partial x^{2}} u(t)\right\|\left\|\frac{\partial^{4}}{\partial x^{4}} u(t)\right\|+\|u(t)\|_{4}^{2}\left\|\frac{\partial^{4}}{\partial x^{4}} u(t)\right\| \\
& +\|h(t)\|\left\|\frac{\partial^{4}}{\partial x^{4}} u(t)\right\|+\left\|\frac{\partial^{2}}{\partial x^{2}} u(t)\right\|^{2} \\
& \leq c\left(\left\|\frac{\partial^{4}}{\partial x^{4}} u(t)\right\|^{\frac{3}{32}}\|u(t)\|^{\frac{29}{32}}\right)^{2}\left(\left\|\frac{\partial^{4}}{\partial x^{4}} u(t)\right\|^{\frac{9}{16}}\|u(t)\|^{\frac{7}{16}}\right)\left\|\frac{\partial^{4}}{\partial x^{4}} u(t)\right\| \\
& +c\left(\left\|\frac{\partial^{4}}{\partial x^{4}} u(t)\right\|^{\frac{1}{8}}\|u(t)\|^{\frac{7}{8}}\right)\left(\left\|\frac{\partial^{4}}{\partial x^{4}} u(t)\right\|^{\frac{5}{16}}\|u(t)\|^{\frac{11}{16}}\right)^{2}\left\|\frac{\partial^{4}}{\partial x^{4}} u(t)\right\| \\
& +\left\|\frac{\partial^{2}}{\partial x^{2}} u(t)\right\|\left\|\frac{\partial^{4}}{\partial x^{4}} u(t)\right\|+c\left(\left\|\frac{\partial^{4}}{\partial x^{4}} u(t)\right\|^{\frac{1}{16}}\|u(t)\|^{\frac{15}{16}}\right)^{2}\left\|\frac{\partial^{4}}{\partial x^{4}} u(t)\right\|
\end{aligned}
$$




$$
\begin{aligned}
& +\|h(t)\|\left\|\frac{\partial^{4}}{\partial x^{4}} u(t)\right\|+\left\|\frac{\partial^{2}}{\partial x^{2}} u(t)\right\|^{2} \\
& \leq \frac{1}{2}\left\|\frac{\partial^{4}}{\partial x^{4}} u(t)\right\|^{2}+c\left(\|u(t)\|^{18}+\|u(t)\|^{\frac{30}{7}}+\left\|\frac{\partial^{2}}{\partial x^{2}} u(t)\right\|^{2}+\|h(t)\|^{2}\right),
\end{aligned}
$$

that is

$$
\begin{aligned}
& \frac{d}{d t}\left\|\frac{\partial^{2}}{\partial x^{2}} u(t)\right\|^{2}+\left\|\frac{\partial^{4}}{\partial x^{4}} u(t)\right\|^{2} \leq c\left(\|u(t)\|^{18}+\|u(t)\|^{\frac{30}{7}}+\left\|\frac{\partial^{2}}{\partial x^{2}} u(t)\right\|^{2}+\|h(t)\|^{2}\right), \\
& \frac{d}{d t}\left\|\frac{\partial^{2}}{\partial x^{2}} u(t)\right\|^{2}+\lambda\left\|\frac{\partial^{2}}{\partial x^{2}} u(t)\right\|^{2} \\
& \leq c\left(\|u(t)\|^{18}+\|u(t)\|^{\frac{30}{7}}+\left\|\frac{\partial^{2}}{\partial x^{2}} u(t)\right\|^{2}+\|h(t)\|^{2}\right) .
\end{aligned}
$$

Multiplying (3.6) by $(t-\tau) e^{\lambda t}$ and integrating it over $(\tau, t)$, we obtain

$$
\begin{aligned}
(t-\tau) e^{\lambda t}\left\|\frac{\partial^{2}}{\partial x^{2}} u(t)\right\|^{2} \leq c\left[\int_{\tau}^{t}(1+(s-\tau)) e^{\lambda s}\left\|\frac{\partial^{2}}{\partial x^{2}} u(s)\right\|^{2} d s\right. \\
\left.\quad+\int_{\tau}^{t}(s-\tau) e^{\lambda s}\|h(s)\|^{2} d s+\int_{\tau}^{t}(s-\tau) e^{\lambda s}\left(\|u(s)\|^{18}+\|u(s)\|^{\frac{30}{7}}\right) d s\right] .
\end{aligned}
$$

Therefore

$$
\begin{aligned}
\left\|\frac{\partial^{2}}{\partial x^{2}} u(t)\right\|^{2} \leq & c\left(1+\frac{1}{t-\tau}\right) e^{-\lambda t} \int_{\tau}^{t} e^{\lambda s}\left\|\frac{\partial^{2}}{\partial x^{2}} u(s)\right\|^{2} d s+c e^{-\lambda t} G_{1}(t) \\
& +c e^{-\lambda t} \int_{\tau}^{t} e^{\lambda s}\|u(s)\|^{18} d s+c e^{-\lambda t} \int_{\tau}^{t} e^{\lambda s}\|u(s)\|^{\frac{30}{7}} d s \\
:= & I_{1}+I_{2}+I_{3}+I_{4} .
\end{aligned}
$$

We have

$$
\begin{aligned}
I_{1}+I_{2} \leq & c\left(1+(t-\tau)+\frac{1}{t-\tau}\right) e^{-\lambda(t-\tau)}\left(\left\|u_{\tau}\right\|_{-1}^{2}+\left\|u_{\tau}\right\|^{2}\right) \\
& +c\left(1+\frac{1}{t-\tau}\right)\left\{1+e^{-\lambda t}\left[G_{1}(t)+G_{2}(t)\right]\right\} .
\end{aligned}
$$

Note that $e^{-8 \lambda(t-\tau)} \leq 1$ for $s \in[\tau, t]$. Thus,

$$
\begin{aligned}
I_{3} \leq & c e^{-\lambda t} \int_{\tau}^{t} e^{\lambda s}\left[e^{-\lambda(s-\tau)}\left(\left\|u_{\tau}\right\|_{-1}^{2}+\left\|u_{\tau}\right\|^{2}\right)+\frac{M_{1}+M_{2}}{\lambda}+\frac{4}{\lambda} e^{-\lambda s} G_{1}(s)\right]^{9} d s \\
\leq & c e^{-\lambda t} \int_{\tau}^{t} e^{\lambda s} d s+c e^{-\lambda t} \int_{\tau}^{t} e^{\lambda s} e^{-9 \lambda(s-\tau)}\left(\left\|u_{\tau}\right\|_{-1}^{2}+\left\|u_{\tau}\right\|^{2}\right)^{9} d s \\
& +c e^{-\lambda t} \int_{\tau}^{t} e^{\lambda s} e^{-9 \lambda s}\left[G_{1}(s)\right]^{9} d s
\end{aligned}
$$




$$
\begin{aligned}
\leq & c e^{-\lambda t}\left(e^{\lambda t}-e^{\lambda \tau}\right)+c e^{-\lambda(t-\tau)}\left(\left\|u_{\tau}\right\|_{-1}^{2}+\left\|u_{\tau}\right\|^{2}\right)^{9} \int_{\tau}^{t} e^{-8 \lambda(s-\tau)} d s \\
& +c e^{-\lambda t} \int_{\tau}^{t} e^{-8 \lambda s}\left[G_{1}(s)\right]^{9} d s \\
\leq & c+c(t-\tau) e^{-\lambda(t-\tau)}\left(\left\|u_{\tau}\right\|_{-1}^{18}+\left\|u_{\tau}\right\|^{18}\right)+c e^{-\lambda t} \int_{\tau}^{t} e^{-8 \lambda s}\left[G_{1}(s)\right]^{9} d s .
\end{aligned}
$$

Using the same method as (3.8), we derive that

$$
I_{4} \leq c+c(t-\tau) e^{-\lambda(t-\tau)}\left(\left\|u_{\tau}\right\|_{-1}^{\frac{30}{7}}+\left\|u_{\tau}\right\|^{\frac{30}{7}}\right)+c e^{-\lambda t} \int_{\tau}^{t} e^{-\frac{8}{7} \lambda s}\left[G_{1}(s)\right]^{\frac{15}{7}} d s .
$$

Combining these estimates with (3.7), we complete the proof.

Suppose that $\mathfrak{R}$ be the set of all function $r: \mathbb{R} \rightarrow(0, \infty)$ such that

$$
\lim _{t \rightarrow-\infty} t e^{\lambda t} r^{18}(t)=0 .
$$

Denote by $\mathcal{D}$ the class of all families $\hat{D}:=\{D(t): t \in \mathbb{R}\} \subset B\left(H_{0}^{2}(\Omega)\right)$ such that $D(t) \subset \overline{B_{0}}(r(t))$ for some $r(t) \in \mathfrak{R}, \overline{B_{0}}(r(t))$ denote the closed ball in $H_{0}^{2}(\Omega)$ with radius $r(t)$. Let

$$
\begin{aligned}
r_{0}^{2}(t)=2 c & {\left[1+e^{-\lambda t}\left(G_{1}(t)+G_{2}(t)+\int_{\tau}^{t} e^{-8 \lambda s}\left[G_{1}(s)\right]^{9} d s\right.\right.} \\
& \left.\left.+\int_{\tau}^{t} e^{-\frac{8}{7} \lambda s}\left[G_{1}(s)\right]^{\frac{15}{7}} d s\right)\right] .
\end{aligned}
$$

Obviously, for any $\hat{D} \in \mathcal{D}$ and $t \in \mathbb{R}$, by the result of Lemma 3 , there exists $\tau_{0}(\hat{D}, t)<t$ such that

$$
\left\|\frac{\partial^{2}}{\partial x^{2}} u(t)\right\| \leq r_{0}(t), \quad \forall \tau<\tau_{0}
$$

Furthermore, since $0 \leq \alpha<\frac{\lambda}{81}$, we get $\overline{B_{0}}\left(r_{0}(t)\right) \in \mathcal{D}$. Therefore, $\overline{B_{0}}\left(r_{0}(t)\right)$ is a family of bounded pullback $\mathcal{D}$-absorbing sets in $H_{0}^{2}(\Omega)$.

Now, we give the proof of Theorem 1.

Proof. In order to prove the existence of pullback attractor for problem (1.3)(1.5), we need only prove that the process $\{u(t, \tau)\}$ is pullback $\omega-\mathcal{D}$-limit compact (PDC).Thanks to $A^{-1}$ is a continuous compact operator in $L^{2}(0,1)$, there exists a sequence $\left\{\lambda_{j}\right\}_{j=1}^{\infty}$ satisfying

$$
\lambda_{1} \leq \lambda_{2} \leq \cdots \leq \lambda_{j} \leq \cdots, \quad \lambda_{j} \rightarrow \infty, \quad \text { as } j \rightarrow \infty
$$

and a family of elements $\left\{w_{j}\right\}_{j=1}^{\infty}$ of $H_{0}^{2}(0,1)$ which are orthonormal in $L^{2}(0,1)$ such that

$$
A w_{j}=\lambda_{j} w_{j}, \quad \text { for } j=1,2, \cdots .
$$


Write $X_{n}=\operatorname{span}\left\{w_{1}, w_{2}, \cdots, w_{n}\right\} \subset H_{0}^{2}(\Omega)$ and $P_{n}: H_{0}^{2}(0,1) \rightarrow X_{n}$ is an orthogonal projector. Hence

$$
u=P_{n} u+\left(I-P_{n}\right) u:=u_{1}+u_{2} .
$$

Taking the scalar product of (1.3) with $\frac{\partial^{4}}{\partial x^{4}} u_{2}(t)$, using Young's inequality, we get

$$
\begin{aligned}
& \frac{d}{d t}\left\|\frac{\partial^{2}}{\partial x^{2}} u_{2}(t)\right\|^{2}+\left\|\frac{\partial^{4}}{\partial x^{4}} u_{2}(t)\right\|^{2} \\
& \quad \leq c\left(\left\|\frac{\partial^{2}}{\partial x^{2}} u_{2}(t)\right\|^{2}+\|h(t)\|^{2}+\|u(t)\|^{18}+\|u(t)\|^{\frac{30}{7}}\right),
\end{aligned}
$$

which means

$$
\begin{aligned}
& \frac{d}{d t}\left\|\frac{\partial^{2}}{\partial x^{2}} u_{2}(t)\right\|^{2}+\left\|\frac{\partial^{4}}{\partial x^{4}} u_{2}(t)\right\|^{2} \\
& \quad \leq c\left(\left\|\frac{\partial^{2}}{\partial x^{2}} u_{2}(t)\right\|^{2}+\|h(t)\|^{2}+\left\|\frac{\partial^{2}}{\partial x^{2}} u(t)\right\|^{18}+\left\|\frac{\partial^{2}}{\partial x^{2}} u(t)\right\|^{\frac{30}{7}}\right) .
\end{aligned}
$$

Multiplying (3.10) by $(t-\tau) e^{\lambda_{n} t}$ and integrating it over $(\tau, t)$, we deduce that

$$
\begin{aligned}
& (t-\tau) e^{\lambda_{n} t}\left\|\frac{\partial^{2}}{\partial x^{2}} u_{2}(t)\right\|^{2} \leq \int_{\tau}^{t} e^{\lambda_{n} s}\left\|\frac{\partial^{2}}{\partial x^{2}} u_{2}(t)\right\|^{2} d s+c(t-\tau) \int_{\tau}^{t} e^{\lambda_{n} s}\|h(s)\|^{2} d s \\
& +c(t-\tau) \int_{\tau}^{t} e^{\lambda_{n} s}\left(\left\|\frac{\partial^{2}}{\partial x^{2}} u_{2}(t)\right\|^{2}+\left\|\frac{\partial^{2}}{\partial x^{2}} u(t)\right\|^{18}+\left\|\frac{\partial^{2}}{\partial x^{2}} u(t)\right\|^{\frac{30}{7}}\right) d s .
\end{aligned}
$$

Then it follows from (3.9) and (3.11) that

$$
\begin{gathered}
\left\|\frac{\partial^{2}}{\partial x^{2}} u_{2}(t)\right\|^{2} \leq(t-\tau)^{-1} e^{-\lambda_{n} t} \int_{\tau}^{t} e^{\lambda_{n} s}\left\|\frac{\partial^{2}}{\partial x^{2}} u_{2}(t)\right\|^{2} d s+c e^{-\lambda_{n} t} \int_{\tau}^{t} e^{\lambda_{n} s}\|h(s)\|^{2} d s \\
\quad+c e^{-\lambda_{n} t} \int_{\tau}^{t} e^{\lambda_{n} s}\left(\left\|\frac{\partial^{2}}{\partial x^{2}} u_{2}(t)\right\|^{2}+\left\|\frac{\partial^{2}}{\partial x^{2}} u(t)\right\|^{18}+\left\|\frac{\partial^{2}}{\partial x^{2}} u(t)\right\|^{\frac{30}{7}}\right) d s \\
\leq c(t-\tau)^{-1} e^{-\lambda_{n} t} \int_{\tau}^{t} e^{\lambda_{n} s} r_{0}^{2}(s) d x+c e^{-\lambda_{n} t} \int_{\tau}^{t} e^{\lambda_{n} s}\|h(s)\|^{2} d s \\
+c e^{-\lambda_{n} t} \int_{\tau}^{t} e^{\lambda_{n} s}\left[r_{0}^{2}(s)+r_{0}^{18}(s)+r_{0}^{\frac{30}{7}}(s)\right] d s, \quad \forall \tau \leq \tau_{0} .
\end{gathered}
$$

Note that

$$
\begin{gathered}
e^{-\lambda_{n} t} \int_{\tau}^{t} e^{\lambda_{n} s} r_{0}^{2}(s) d s=c e^{-\lambda_{n} t} \int_{\tau}^{t} e^{\lambda_{n} s}\left[1+e^{-\lambda s}\left(G_{1}(s)+G_{2}(s)\right.\right. \\
\left.\quad+\int_{\tau}^{s} e^{-8 \lambda r}\left[G_{1}(r)\right]^{9} d s+\int_{\tau}^{s} e^{-\frac{8}{7} \lambda r}\left[G_{1}(r)\right]^{\frac{15}{7}} d s\right] d r \\
\leq c \lambda_{n}^{-1}+c\left(\lambda_{n}-\lambda\right)^{-1} e^{-\lambda t}\left[G_{1}(t)+G_{2}(t)\right]
\end{gathered}
$$




$$
+c\left(\lambda_{n}-\lambda\right)^{-1} e^{-\lambda t}\left[\int_{-\infty}^{t} e^{-8 \lambda s}\left[G_{1}(s)\right]^{9} d s+\int_{-\infty}^{t} e^{-\frac{8}{7} \lambda s}\left[G_{1}(s)\right]^{\frac{15}{7}} d s\right] .
$$

Similarly, applying $(a+b)^{p} \leq c\left(a^{p}+b^{p}\right)$, we derive that

$$
\begin{aligned}
& e^{-\lambda_{n} t} \int_{\tau}^{t} e^{\lambda_{n} s} r_{0}^{18}(s) d s \leq c \lambda_{n}^{-1}+c\left(\lambda_{n}-9 \lambda\right)^{-1} e^{-9 \lambda t}\left\{\left[G_{1}(t)\right]^{9}+\left[G_{2}(t)\right]^{9}\right\} \\
& +c\left(\lambda_{n}-9 \lambda\right)^{-1} e^{-9 \lambda t}\left[\int_{-\infty}^{t} e^{-8 \lambda s}\left[G_{1}(s)\right]^{9} d s+\int_{-\infty}^{t} e^{-\frac{8}{7} \lambda s}\left[G_{1}(s)\right]^{\frac{15}{7}} d s\right]^{9}
\end{aligned}
$$

and

$$
\begin{aligned}
& e^{-\lambda_{n} t} \int_{\tau}^{t} e^{\lambda_{n} s} r_{0}^{\frac{30}{7}}(s) d s \leq c \lambda_{n}^{-1}+c\left(\lambda_{n}-\frac{15}{7} \lambda\right)^{-1} e^{-\frac{15}{7} \lambda t}\left\{\left[G_{1}(t)\right]^{\frac{15}{7}}+\left[G_{2}(t)\right]^{\frac{15}{7}}\right\} \\
& +c\left(\lambda_{n}-\frac{15}{7} \lambda\right)^{-1} e^{-\frac{15}{7} \lambda t}\left[\int_{-\infty}^{t} e^{-8 \lambda s}\left[G_{1}(s)\right]^{9} d s+\int_{-\infty}^{t} e^{-\frac{8}{7} \lambda s}\left[G_{1}(s)\right]^{\frac{15}{7}} d s\right]^{\frac{15}{7}}
\end{aligned}
$$

On the other hand, simple calculations show that

$$
\begin{aligned}
& e^{-\lambda_{n} t} \int_{\tau}^{t} e^{\lambda_{n} s}\|h(s)\|^{2} d s \\
& \leq\left\{\begin{array}{l}
\beta e^{-\lambda_{n} t} \int_{\tau}^{t} e^{\lambda_{n} s} e^{-\alpha s} d s, \quad t \leq 0, \\
\beta e^{-\lambda_{n} t} \int_{\tau}^{t} e^{\lambda_{n} s} e^{\alpha|s|} d s, \quad t \geq 0,
\end{array} \leq\left\{\begin{array}{l}
\frac{\beta e^{-\lambda_{n} t}}{\lambda_{n}-\alpha}, \quad t \leq 0, \\
\frac{\beta e^{-\lambda_{n} t}}{\lambda_{n}-\alpha}+\frac{\beta e^{\alpha t}}{\lambda_{n}+\alpha}, \quad t \geq 0 .
\end{array}\right.\right.
\end{aligned}
$$

Adding (3.12)-(3.16) together, we can obtain for any $\varepsilon>0$, there exist $\tau_{0}<t$ and $N \in \mathbb{N}$ such that

$$
\left\|\Delta u_{2}(t)\right\| \leq \varepsilon, \quad \forall \tau<\tau_{0} .
$$

This infer that the process $\{U(t, \tau)\}$ is pullback $\omega-\mathcal{D}$-limit compact. Then, by Lemma 1 , the process corresponding to problem (1.3)-(1.5) possesses a unique pullback $\mathcal{D}$-attractor in $H_{0}^{2}(\Omega)$.

Remark 2. In this article, based on the techniques in $[17,20]$, we study the existence of pullback attractor for a non-autonomous generalized Cahn-Hilliard equation in $1 \mathrm{D}$ case. It seems that the study on the following non-autonomous equation

$$
\frac{\partial u}{\partial t}+\varepsilon^{2} \Delta^{2} u-\Delta f(u)+g(u)=h(x, t), \quad x \in \mathbb{R}^{N}, t \in[\tau, \infty), \quad N \geq 2
$$

are more important and difficult. However, so far by now, we could not obtain satisfactory result and just leave them as problems to be carried out later.

\section{Acknowledgements}

The author would like to express her deep thanks to the referee's valuable suggestions for the revision and improvement of the manuscript. 


\section{References}

[1] A. Bonfoh. Finite-dimensional attractor for the viscous Cahn-Hilliard equation in an unbounded domain. Quarterly of Applied Mathematics, 64:93-104, 2006. http://dx.doi.org/10.1090/S0033-569X-06-00988-3.

[2] J.W. Cahn and J.E. Hilliard. Free energy of a nonuniform system. I. interfacial free energy. The Journal of Chemical Physics, 28(2):258-267, 1958. http://dx.doi.org/10.1063/1.1744102.

[3] T. Caraballo and R. Colucci. Pullback attractor for a non-linear evolution equation in elasticity. Nonlinear Analysis: Real World Applications, 15:80-88, 2014. http://dx.doi.org/10.1016/j.nonrwa.2013.06.002.

[4] T. Caraballo, G. Kiss and T. Taniguchi. Pullback attractors for differential equations with multiple variable delays in Lipschitz nonlinearities. International Journal of Bifurcation and Chaos, 23(11):1350187, 2013. http://dx.doi.org/10.1142/S0218127413501873.

[5] T. Caraballo, G. Łukaszewicz and J. Real. Pullback attractors for asymptotically compact non-autonomous dynamical systems. Nonlinear Analysis: Theory, Methods \& Applications, 64(3):484-498, 2006. http://dx.doi.org/10.1016/j.na.2005.03.111.

[6] L. Cherfils, A. Miranville and S. Zelik. The Cahn-Hilliard equation with logarithmic potentials. Milan Journal of Mathematics, 79(2):561-596, 2011. http://dx.doi.org/10.1007/s00032-011-0165-4.

[7] L. Cherfils, A. Miranville and S. Zelik. On a generalized Cahn-Hilliard equation with biological applications. Discrete and Continuous Dynamical Systems - Series B, 19(7):2013-2026, 2014. http://dx.doi.org/10.3934/dcdsb.2014.19.2013.

[8] D.S. Cohen and J.D. Murray. A generalized diffusion model for growth and dispersal in a population. Journal of Mathematical Biology, 12(2):237-249, 1981. http://dx.doi.org/10.1007/BF00276132.

[9] C.M. Elliott and S. Zheng. On the Cahn-Hilliard equation. Archive for Rational Mechanics and Analysis, 96:339-357, 1986. http://dx.doi.org/10.1007/BF00251803.

[10] J. Fort and V. Méndez. Time-delayed theory of the neolithic transition in Europe. Physical Review Letters, 82:867-870, 1999. http://dx.doi.org/10.1103/PhysRevLett.82.867.

[11] J. Fort and V. Méndez. Time-delayed spread of viruses in growing plaques. Physical Review Letters, 89(17):178101, 2002. http://dx.doi.org/10.1103/PhysRevLett.89.178101.

[12] P. Galenko and D. Jou. Diffuse-interface model for rapid phase transformations in nonequilibrium systems. Physical Review E, 71(4):046125, 2005. http://dx.doi.org/10.1103/PhysRevE.71.046125.

[13] P. Galenko and V. Lebedev. Analysis of the dispersion relation in spinodal decomposition of a binary system. Philosophical Magazine Letters, 87(11):821827, 2007. http://dx.doi.org/10.1080/09500830701395127.

[14] G. Gilardi, A. Miranville and G. Schimperna. On the Cahn-Hilliard equation with irregular potentials and dynamic boundary conditions. Commun. Pure and Applied Anal., 8(3):881-912, 2009. http://dx.doi.org/10.3934/cpaa.2009.8.881. 
[15] M. Grasselli, G. Schimperna and S. Zelik. On the 2D Cahn-Hilliard equation with inertial term. Communications in Partial Differential Equation, 34(2):137-170, 2009. http://dx.doi.org/10.1080/03605300802608247.

[16] E. Khain and L.M. Sander. Generalized Cahn-Hilliard equation for biological applications. Physical Review E, 77(5):051129, 2008. http://dx.doi.org/10.1103/PhysRevE.77.051129.

[17] Y. Li and C.K. Zhong. Pullback attractors for the norm-to-weak continuous process and application to the nonautonomous reaction-diffusion equations. Applied Mathematics and Computation, 190(2):1020-1029, 2007. http://dx.doi.org/10.1016/j.amc.2006.11.187.

[18] B. Liu and C.V. Pao. Integral representation of generalized diffusion model in population problems. Journal of Integral Equation, 6(2):175-185, 1984.

[19] A. Miranville. Asymptotic behaviour of a generalized Cahn-Hilliard equation with a proliferation term. Applicable Analysis, 92(6):1308-1321, 2013. http://dx.doi.org/10.1080/00036811.2012.671301.

[20] S.H. Park and J.Y. Park. Pullback attractor for a non-autonomous modified Swift-Hohenberg equation. Computers \& Mathematics with Applications, 67(3):542-548, 2014. http://dx.doi.org/10.1016/j.camwa.2013.11.011.

[21] G. Schimperna. Global attractors for Cahn-Hilliard equations with nonconstant mobility. Nonlinearity, 20:2365-2387, 2007. http://dx.doi.org/10.1088/0951$7715 / 20 / 10 / 006$.

[22] A. Segatti. On the hyperbolic relaxation of the Cahn-Hilliard equation in 3D: approximation and long time behaviour. Math. Models Methods Appl. Sci., 17(03):411-437, 2007. http://dx.doi.org/10.1142/S0218202507001978.

[23] H. Song and H. Wu. Pullback attractors of nonautonomous reaction-diffusion equations. Journal of Mathematical Analysis and Applications, 325(2):12001215, 2007. http://dx.doi.org/10.1016/j.jmaa.2006.02.041.

[24] R. Temam. Infinite-Dimensional Dynamical Systems in Mechanics and Physics. Applied Mathematical Sciences. Springer US, New York, 1988. http://dx.doi.org/10.1007/978-1-4684-0313-8.

[25] X. Zhao, N. Duan and B. Liu. Optimal control problem of a generalized Ginzburg-Landau model equation in population problems. Mathematical Methods in the Applied Sciences, 37(3):435-446, 2014. http://dx.doi.org/10.1002/mma.2806. 